\title{
Expression of TRPC5 is decreased in the sperm of patients with varicocele-associated asthenozoospermia
}

\author{
GUANGBIN ZHU ${ }^{1,2^{*}}$, CHANGYING XIE ${ }^{1 *}$, ZHONGHUA YANG $^{2}$, YONGZHI WANG $^{2}$, \\ DONG CHEN $^{2}$ and XINGHUAN WANG ${ }^{2}$ \\ ${ }^{1}$ Department of Urology, Daqing Oilfield General Hospital, Daqing, Heilongjiang 163000; ${ }^{2}$ Department of Urology, \\ Zhongnan Hospital of Wuhan University, Wuhan, Hubei 430071, P.R. China
}

Received November 2, 2017; Accepted March 28, 2018

DOI: $10.3892 /$ br.2018.1089

\begin{abstract}
The present study aimed to determine whether the expression of transient receptor potential channel 5 (TRPC5) protein is altered in spermatozoa of patients with varicocele-associated asthenozoospermia. TRPC5 expression in spermatozoa was determined by polymerase chain reaction and western blotting analyses, and indirect immunofluorescence was used for identification and immunolocalization of the TRPC5 channel in human sperm. Sperm motility and superoxide dismutase (SOD) activity were also determined with a computer-assisted semen analysis system and assay kit, respectively. Compared with levels in control subjects, it was identified that TRPC5 protein expression, SOD activity and cellular motility in the sperm of patients with varicocele-associated asthenozoospermia were reduced $(\mathrm{P}<0.001)$. Furthermore, the expression of TRPC5 was positively correlated with sperm motility $(\mathrm{r}=0.781, \mathrm{P}<0.001)$ and SOD activity $(\mathrm{r}=0.933, \mathrm{P}<0.001)$, indicated by partial correlation analysis. The present study may provide a novel target for the study and treatment of varicocele-associated asthenozoospermia.
\end{abstract}

\section{Introduction}

Fertilization is the phenomenon in which sperm and egg locate each other, interact and fuse. This phenomenon is essential for all plants and animals that engage in sexual reproduction. A previous study on mammalian sperm indicated that an increase in the concentration of intracellular $\mathrm{Ca}^{2+}\left[\left(\mathrm{Ca}^{2+}\right)_{\mathrm{i}}\right]$ was critical for several physiological processes during fertilization,

Correspondence to: Professor Xinghuan Wang, Department of Urology, Zhongnan Hospital of Wuhan University, 169 Donghu Road, Wuhan, Hubei 430071, P.R. China

E-mail: 3051761@qq.com

*Contributed equally

Key words: transient receptor potential channel, male infertility, sperm, $\mathrm{Ca}^{2+}$ channel, varicocele, asthenozoospermia including sperm motility, capacitation, and acrosome reaction (AR) (1). Furthermore, calcium has been demonstrated to serve roles in initiation of motility, capacitation and AR in spermatozoa, as reviewed by Kumar and Shoeb (2).

A number of calcium channels including homologues of the Drosophila transient receptor potential (TRP) proteins have been identified in sperm (3). TRP channels are a superfamily of functionally versatile cation permeation channels that are present in most mammalian cell types. As molecular sensors of the external environment, the TRP ion channels may serve an important role in sperm maturation and development of fertilization potential $(4,5)$.

Initially proposed as store-operated calcium channels in mammals, products of the seven TRPC genes (TRPC1-7) have been identified based on sequence similarities to TRP $(6,7)$, and have been implicated in an increasing range of physiological processes (8-10). These $\mathrm{Ca}^{2+}$ entry pathways are also present in mammalian sperm and control the initial stages of fertilization (11). Although the function of the different TRPC channels in the sperm flagellum is unknown, it has been speculated that they may participate in key events required for fertilization, such as the initiation of motility (activation) and hyperactivation (12).

In a previous study, Castellano et al (13) first detected TRPC1, 3, 4, 6 and 7 in human sperm and indicated that these $\mathrm{Ca}^{2+}$-permeable channels may influence flagellar movement; however, because of the lack of specific antibody, they could not detect the TRPC5 channel. Nevertheless, the association between sperm TRPC channels and human male infertility is unknown at present.

Infertility is commonly defined as the failure of conception after $\geq 12$ months of unprotected intercourse (14). Statistics indicate that approximately $15 \%$ of all couples in the United States are infertile, and that the male factor is responsible for $\sim 25 \%$ of these cases $(15,16)$. Varicocele is the most frequent abnormal physical finding in men who present for infertility evaluation, and represents the most common treatable cause of male factor infertility (17). Varicocele is associated with impairment in spermatogenesis in the form of asthenozoospermia, which is defined by the proportion of motile spermatozoa in semen (grade a+b sperm motility, $<50 \%$; or grade a, $<25 \%$ ) according to the 2010 World Health Organization laboratory manual for the examination and processing of human semen (18). 
As the expression of sperm TRPCs channels is involved in the regulation of flagellar motility in human sperm, the present study investigated the expression of TRPC channels in spermatozoa from infertile men, which, to the best of our knowledge, has not been reported to date. For this purpose, the expression of TRPC5 from ejaculated spermatozoa was compared between patients with varicocele-associated asthenozoospermia and age-matched control subjects.

\section{Materials and methods}

Ejaculates. The study population consisted of 40 patients with varicocele-associated asthenozoospermia and 40 age-matched control healthy subjects with normal semen parameters from the Department of Andrology of Zhongnan Hospital, Wuhan University (Wuhan, China). The demographic characteristics of subjects in each group are listed in Table I. Seminal samples were obtained by masturbation after 3-5 days of sexual abstinence. Patients with pyospermia were excluded. It was not assessed whether patients had experienced a recent fever or toxicant exposure or were smokers. Informed consent was obtained from subjects permitting the use of their samples and data for the current study purposes. The study protocol was approved by the Ethics Committee of Zhongnan Hospital of Wuhan University.

Evaluation of sperm. Samples in individual glass containers were kept at ambient temperature between 20 and $37^{\circ} \mathrm{C}$. Sperm from each subject was divided into two samples. For liquefaction, one sample from each subject was placed in an incubator at $37^{\circ} \mathrm{C}$. Following liquefaction, sperm were tracked and analyzed with a computer-assisted semen analysis (CASA) system (CASAS-QH-III; Beijing Huafang Shenhuo Technology Co., Ltd., Beijing, China). The following sperm parameters were assessed: Ejaculate volume, $\mathrm{pH}$, density and motility.

Percoll density gradient separation. Percoll density gradient separation was performed as described by Parrish et al (19) with some modifications. A stock of Percoll solution (Sigma-Aldrich; Merck KGaA, Darmstadt, Germany) was prepared at a 9:1 mixture of Percoll and x10 stock of salt solution (2.889 $\mathrm{g} \mathrm{NaCl}, 0.238 \mathrm{~g} \mathrm{KCl}, 0.116 \mathrm{~g} \mathrm{KH}_{2} \mathrm{PO}_{4}, 0.112 \mathrm{~g} \mathrm{CaCl}_{2}$ and $0.163 \mathrm{~g}$ Hepes in $50 \mathrm{ml}$ distilled water). A 90\% Percoll solution was obtained by diluting the Percoll solution stock with fetal bovine serum (Gibco; Thermo Fisher Scientific, Inc., Waltham, MA, USA) To prepare a $45 \%$ Percoll solution, the $90 \%$ Percoll solution was mixed at a $1: 1$ ratio with $\mathrm{BO}$ medium. In a $15 \mathrm{ml}$ conical tube, $1.5 \mathrm{ml}$ of the $90 \%$ Percoll solution was placed, onto which $1.5 \mathrm{ml}$ of $45 \%$ Percoll was layered. The sperm suspension, collected as described above, was loaded onto the two-step Percoll gradient. Then, semen was added to the Percoll solution. Percoll density gradient separation was performed by centrifugation $(300 \mathrm{x} \mathrm{g}, 20 \mathrm{~min}$, $25^{\circ} \mathrm{C}$ ); the pellet was recovered following aspiration of the supernatant. All recovered semen samples were mixed with $6 \mathrm{ml} \mathrm{BO}$ medium by centrifugation $\left(300 \mathrm{x} \mathrm{g}, 5 \mathrm{~min}, 25^{\circ} \mathrm{C}\right)$.

RNA extraction and reverse transcription-polymerase chain reaction $(R T-P C R)$. Total RNA was isolated from the ejaculates of donors using an RNeasy Mini kit (cat. no. 74104; Qiagen GmbH, Hilden, Germany) and cDNA was synthesized using a SuperScript First-Strand Synthesis system (Invitrogen; Thermo Fisher Scientific, Inc.) for RT-PCR according to the manufacturer's instructions. Specific primers for the TRPC5 channel were designed based on the coding sequence and were as follows (20): Forward, 5'-CTAACTGCCTTCCGTCTG-3' and reverse, 5'-CTAACTGCCTTCCGTCTG-3'. GAPDH was used as the internal control gene, for which the primer sequences were as follows: Forward, 5'-AACGGATTTGGTCGTATTG-3' and reverse, 5'-AATCCCATCACCATCTTCC-3'. The PCR temperature profile was 35 cycles of amplification $\left(94^{\circ} \mathrm{C}\right.$ for $1 \mathrm{~min}, 55^{\circ} \mathrm{C}$ for $1 \mathrm{~min}$ and $72^{\circ} \mathrm{C}$ for $30 \mathrm{sec}$ ) and a final extension at $72^{\circ} \mathrm{C}$ for $3 \mathrm{~min}$. PrimeScript TM RTase (Takara Biotechnology Co., Ltd., Dalian, China) was used as the polymerase. The PCR products were separated on $1 \%$ agarose gels (5 $\mu \mathrm{g} / \mathrm{ml}$ ethyl bromide), purified (GenElute ${ }^{\mathrm{TM}}$ PCR Clean-Up kit; Sigma-Aldrich; Merck KGaA) and sent to Takara Biotechnology Co., Ltd. for sequencing. Sequence identities were established by searching databases in the NCBI Basic Local Alignment Search Tool program (https://blast.ncbi.nlm. nih.gov).

Indirect immunofluorescence. Immunolocolization of the TRPC5 channel in sperm was assessed by fluorescence microscopy. In brief, sperm were fixed in $4 \%$ paraformaldehyde in phosphate-buffered-saline (PBS) for $15 \mathrm{~min}$ at room temperature, then seeded onto polylysine-coated glass slides coated with gelatin bioadhesive (Sigma-Aldrich; Merck KGaA). Cells were permeabilized with $0.2 \%$ Triton X-100 in PBS for $10 \mathrm{~min}$ and blocked for $1 \mathrm{~h}$ in $5 \%$ bovine serum albumin (Procell, Wuhan, China) at room temperature. Samples were then incubated overnight at $4^{\circ} \mathrm{C}$ with anti-TRPC5 antibody (cat. no. sc-293259; Santa Cruz Biotechnology, Inc., La Jolla, CA, USA) at a 1:100 dilution. Samples were subsequently incubated for $1 \mathrm{~h}$ at $37^{\circ} \mathrm{C}$ with fluorescein isothiocyanate-conjugated anti-goat secondary antibody (cat. no. A23230; Jackson Immuno Research Laboratories, Inc., West Grove, PA, USA). Sperm were examined by confocal fluorescence and phase contrast microscopy.

Western blot analysis. Human spermatozoa were pooled and lysed $\left(0^{\circ} \mathrm{C}, 30 \mathrm{~min}\right)$ in lysis buffer $(50 \mathrm{mmol} / 1 \mathrm{Tris}-\mathrm{HCl}, \mathrm{pH} 7.4$, $5 \mathrm{mmol} / 1$ ethylenedi-aminetetraacetic acid, $1 \mathrm{mmol} / 1$ EGTA, $10 \mathrm{mmol} / 1$ 2-mercaptoethanol) containing protease inhibitors $(5 \mu \mathrm{g} / \mathrm{ml}$ leupeptin, $5 \mu \mathrm{g} / \mathrm{ml}$ aprotinin, $10 \mu \mathrm{g} / \mathrm{ml}$ soybean trypsin inhibitor and $1 \mathrm{mmol} / \mathrm{l}$ phenylmethylsulfonyl fluoride) and were subsequently sonicated (power 300-350 W, working $9.9 \mathrm{sec}$, intermittent $9.9 \mathrm{sec}, 152$ cycles). Following centrifugation at $12,000 \mathrm{x} \mathrm{g}$ for $15 \mathrm{~min}$ in $4^{\circ} \mathrm{C}$, the supernatant was recovered, and the total protein content was measured using a bicinchoninic acid kit. The protein expression of TRPC5 and $\beta$-actin was assayed by western blot analysis. Each lane was loaded with $50 \mu \mathrm{g}$ protein, with the separation and spacer gels at 10 and 5\%, respectively. The proteins were transferred to polyvinylidene difluoride membranes, which were blocked in 5\% skimmed milk. Primary incubation was with the anti-TRPC5 antibody (dilution 1:200) with $\beta$-actin (dilution 1:5,000; cat. no. A1978; Sigma-Aldrich; Merck KGaA) used as the loading control. Membranes were incubated in the primary 
Table I. Demographic and clinical data of subjects.

\begin{tabular}{lccr}
\hline Parameter & Controls & Asthenozoospermia & P-value \\
\hline $\mathrm{n}$ & 40 & 40 & 0.778 \\
Age (years) & $33.50 \pm 3.84$ & $32.13 \pm 3.00$ & 0.862 \\
$\mathrm{pH}$ & $7.55 \pm 0.18$ & $7.55 \pm 0.19$ & 0.424 \\
Ejaculate volume $(\mathrm{ml})$ & $3.45 \pm 0.99$ & $3.28 \pm 0.96$ & 0.906 \\
Sperm density $\left(10^{6} / \mathrm{ml}\right)$ & $56.92 \pm 14.87$ & $57.35 \pm 16.69$ & $<0.01$ \\
Grade a+b sperm $(\%)$ & $52.22 \pm 1.33$ & $19.32 \pm 4.53$ & \\
\hline
\end{tabular}

antibody solution with continuous shaking at $4^{\circ} \mathrm{C}$ overnight. Then, the membranes were incubated with corresponding secondary antibodies (dilution 1:2,000; cat. no. ab6808; Abcam, Cambridge, UK) with continuous shaking at room temperature for $1 \mathrm{~h}$. Finally, protein bands were visualized with an Enhanced Chemiluminescence Western Blotting Substrate kit (BioVision, Inc., Milpitas, CA, USA). GraphPad Prism 5 (GraphPad Software, Inc., La Jolla, CA, USA) was used for densitometric analysis.

Determination of superoxide dismutase (SOD) activity in spermatozoa. Sperm SOD activity was measured in spermatozoa using a SOD assay kit (Nanjing Jiancheng Bioengineering Institute, Nanjing, China) according to the manufacturer's instructions. SOD activity was measured by assessing the degree of inhibition of the reduction of nitroblue tetrazolium by xanthine oxidase when specimens were added. Sperm SOD activity was expressed as $\mathrm{U} / \mathrm{mg}$ total protein.

Statistical analysis. The data are presented as the mean \pm standard deviation. For comparison between two independent variables, the Student's unpaired t-test and partial correlation analysis were used. Statistical analysis was performed using SPSS software version 17.0 (SPSS, Inc., Chicago, IL, USA). Statistical significance was considered at $\mathrm{P}<0.05$ for all analyses.

\section{Results}

TRPC5 is expressed in human sperm and downregulated in patients with varicocele-associated asthenozoospermia. First, the presence of TRPC5 in human sperm was verified. A representative agarose gel with ethidium bromide staining of the PCR product of TRPC5 from human ejaculate cDNA is depicted in Fig. 1; the PCR product for TRPC5 has a size of $247 \mathrm{bp}$. The identity of this product was subsequently confirmed by sequencing, which indicated that the TRPC5 gene is expressed in human sperm (data not shown). Next, antibody against TRPC5 was used to investigate its expression at the protein level in human sperm by fluorescence microscopy. Fig. 2 shows representative confocal IF images of human sperm treated with antibody directed against the TRPC5 protein. Of note, the main segment of the flagellum and head were stained to a high level and exhibited uniform fluorescence, indicating uniform distribution of TRPC5 protein on the sperm membrane.

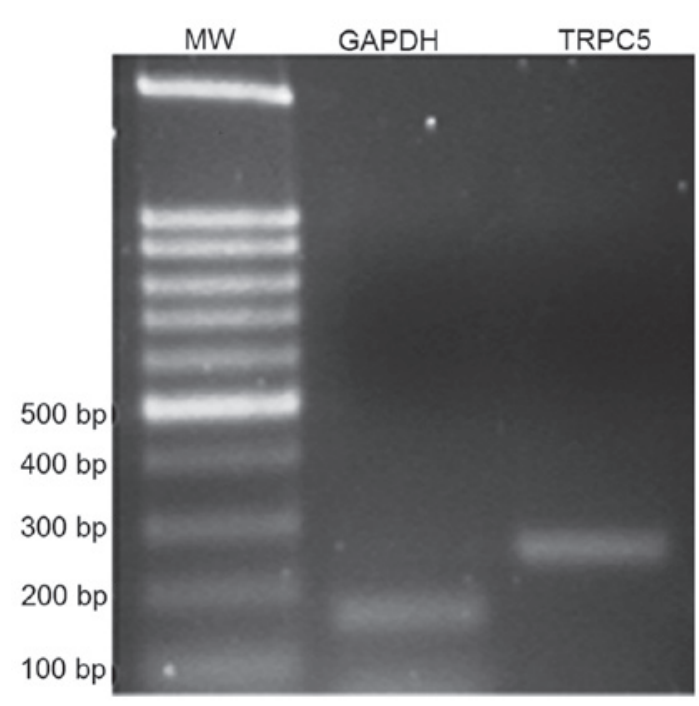

Figure 1. Reverse-transcription polymerase chain reaction analysis of TRPC5 mRNAs expression in human sperm. TRPC5, transient receptor potential channel 5; MW, DNA molecular weight ladder.
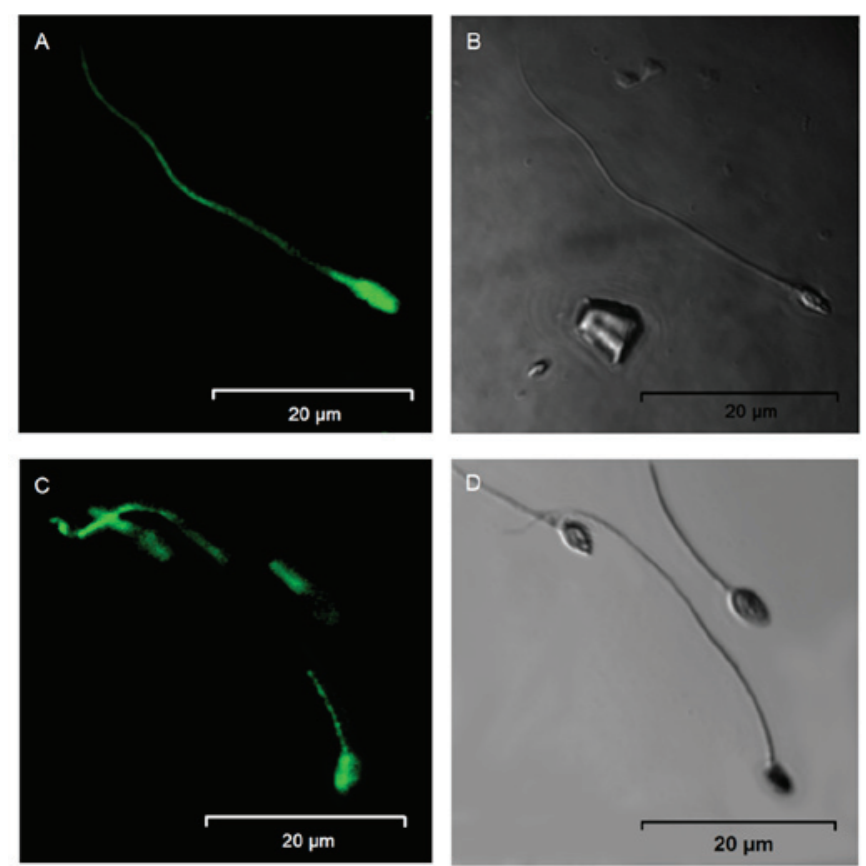

Figure 2. Transient receptor potential channel 5 protein localized in the head and flagellum of human sperm. (A-D) Representative paired photomicrographs are shown: (B and D) Spermatozoa under light microscopy; (A and C) fluorescence view of the same fields. Magnification, x400. 
A

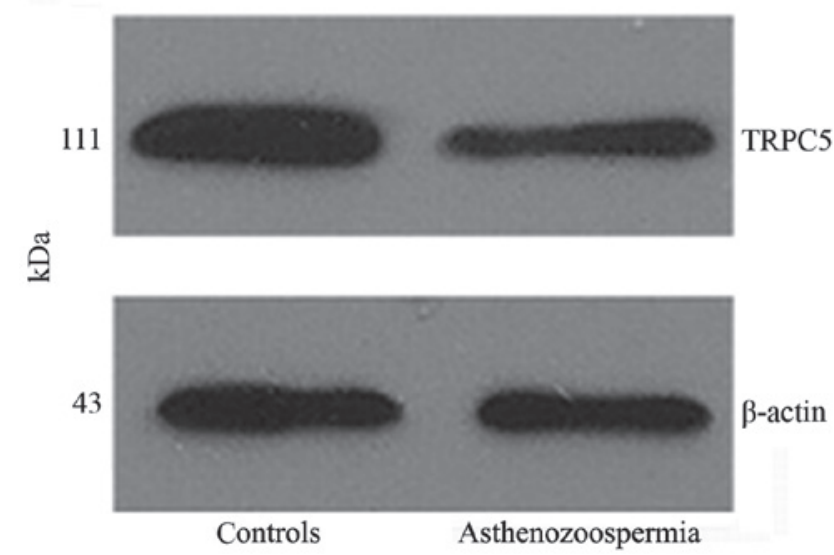

B

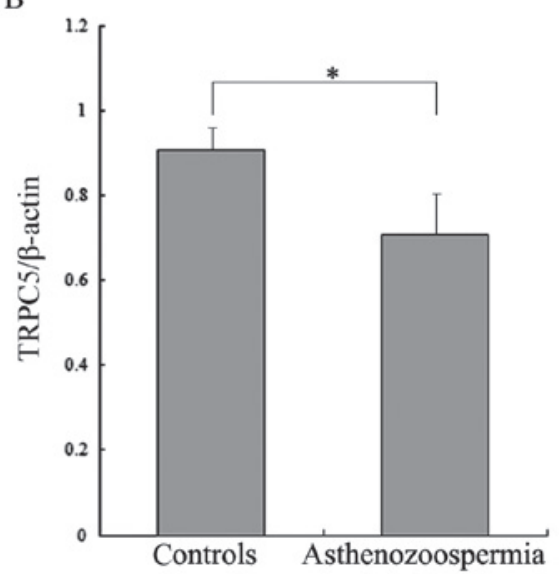

Figure 3. Western blot analysis of TRPC5 expression in sperm from control subjects and patients with asthenozoospermia. (A) TRPC5 expression detected by western blotting. (B) Levels of TRPC5 were normalized against $\beta$-actin expression. ${ }^{*} \mathrm{P}<0.001$ by Student's unpaired t-test. TRPC5, transient receptor potential channel 5 .

Table II. Correlations between the protein expression of TRPC5 and the motility and SOD activity of sperm.

\begin{tabular}{lcc}
\hline & Grade $\mathrm{a}+\mathrm{b}$ sperm (\%) & SOD activity \\
\hline Expression of TRPC5 & $\mathrm{P}<0.001$ & $\mathrm{P}<0.001$ \\
& $\mathrm{r}=0.781$ & $\mathrm{r}=0.933$ \\
\hline
\end{tabular}

TRPC5, transient receptor potential channel 5; SOD, superoxide dismutase.

Furthermore, the presence of the TRPC5 protein was determined by western blot analysis, using specific antibody against TRPC5. The TRPC5 protein was successfully detected in total protein isolate from human spermatozoa. The western blot analysis detected TRPC5 as a single band at $\sim 111 \mathrm{kDa}$ (Fig. 3A). Quantification of the TRPC5 bands revealed that TRPC5 expression was reduced in patients with varicocele-associated asthenozoospermia, compared with that in control subjects $(\mathrm{P}<0.001$; Fig. 3B).

Sperm motility is significantly reduced in patients with varicocele-associated asthenozoospermia. Relevant semen parameters including sperm motility were evaluated using a CASA system. The semen characteristics of the study populations are presented in Table I. Notably, the sperm motility (percentage of grade $a+b$ sperm) was significantly reduced in the patients with asthenozoospermia relative to that in the control subjects $(\mathrm{P}<0.01)$.

SOD activity in spermatozoa is decreased in patients with varicocele-associated asthenozoospermia. The SOD activity of spermatozoa from patients with varicocele-associated asthenozoospermia and control subjects was assessed. The results demonstrated that the mean sperm SOD activity in the asthenozoospermia patients $(84.58 \pm 19.55 \mathrm{U} / \mathrm{mg}$ protein) was significantly lower when compared with that in the control subjects (123.90 $\pm 19.53 \mathrm{U} / \mathrm{mg}$ protein; $\mathrm{P}<0.001$; Fig. 4).

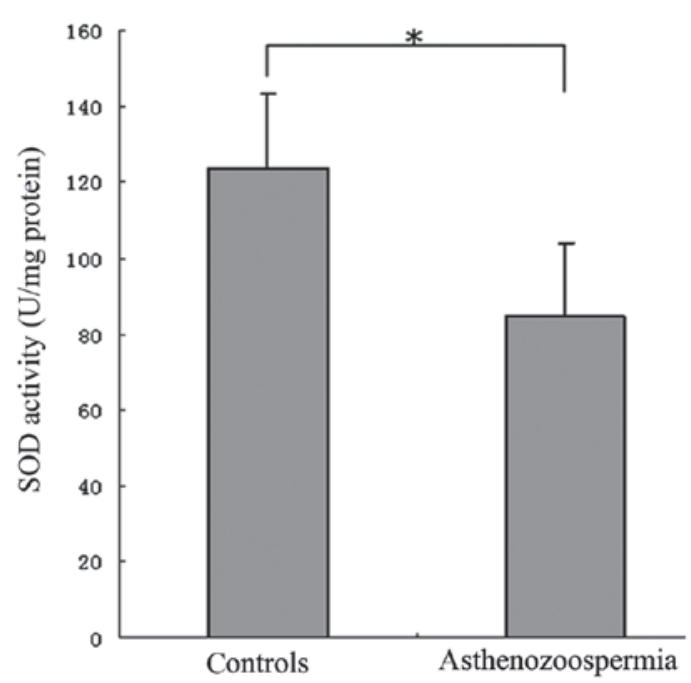

Figure 4. Sperm SOD activity in control subjects and patients with varicocele-associated asthenozoospermia. ${ }^{*} \mathrm{P}<0.001$ by Student's unpaired t-test. SOD, superoxide dismutase.

TRPC5 levels are positively correlated with sperm motility and SOD activity. The level of TRPC5 expression was quantified with GraphPad software. A partial correlation analysis controlling for age was performed to analyze the correlations between the levels of TRPC5 protein in sperm and the percentage of progressively motile (grade $a+b)$ spermatozoa and sperm SOD activity. The results indicated that sperm TRPC5 expression was positively correlated with the percentage of progressively motile spermatozoa $(\mathrm{r}=0.781, \mathrm{P}<0.001)$ and sperm SOD activity $(r=0.933, \mathrm{P}<0.001$; Table II).

\section{Discussion}

In the present study, it was demonstrated, to the best of our knowledge for the first time, that the TRPC5 channel is localized to human sperm, that TRPC5 levels in patients with varicocele-associated asthenozoospermia are reduced compared with those in control subjects, and that sperm TRPC5 levels are positively correlated with sperm motility 
and SOD activity. In addition, the localization of the TRPC5 channel was confirmed by indirect IF. The immunocytochemistry results were consistent with the data of the PCR and western blot analyses as TRPC5 was detected at the RNA and protein levels. The immunoconfocal images demonstrated that TRPC5 was not only present in the sperm head, where it may be involved in sperm AR, but also in the flagellum, suggesting that it may serve to regulate key $\mathrm{Ca}^{2+}$-dependent events in this region including flagellar beating.

Darszon et al (1) indicated in their previous study that an increase in the concentration of $\left(\mathrm{Ca}^{2+}\right)_{\mathrm{i}}$ was critical for several physiological processes during mammalian fertilization, including sperm motility, capacitation and AR. Though different types of voltage-gated $\mathrm{Ca}^{2+}$ channels are present in sperm flagellum and are likely to contribute to the rise in $\left(\mathrm{Ca}^{2+}\right)_{\mathrm{i}}(21)$, TRP proteins may also participate in this event.

The TRP proteins are a novel and expanding superfamily, encompassing non-voltage-gated $\mathrm{Ca}^{2+}$ channels involved in long-lasting $\mathrm{Ca}^{2+}$ entry (2). The TRPC subfamily is composed of proteins that are most highly related to Drosophila TRP proteins (2). TRPCs are widely expressed in the nervous system as well as in non-excitable cells, and serve fundamental roles in processes ranging from sensory physiology to male fertility (13).

To date, the exact function of different TRPCs in the sperm flagellum has not been elucidated; however, it has been speculated that these channels may participate in important events required for fertilization, such as activation and hyperactivation (12). It is established that $\mathrm{Ca}^{2+}$, acting directly on the flagellum axoneme, is a key regulator of sperm motility and hyperactivation (22).

The spermatozoa travel a substantial distance to reach the oocyte, and therefore sperm motility is an important requirement for normal fertilization. Castellano et al (13) reported that TRPCs in human spermatozoa were mainly localized in the flagellum, to provide the motile force necessary for the sperm to reach the oocyte. In addition, their results indicated that the $\mathrm{Ca}^{2+}$-permeable channels may influence flagellar movement (13). However, due to the lack of specific antibody, they could not detect the TRPC5 channel (13). Nonetheless, in their study, sperm motility was potently inhibited by SKF96365, which has been used as a blocker of TRPC5 in a previous study (23). Coupled with the present results, it may be suggested that TRPC5 serves an important role in flagellar movement.

The prevalence of varicocele in the general population is $15-20 \%$, though this is notably greater $(25-40 \%)$ in infertile couples with male factor infertility $(24,25)$. The exact pathophysiological mechanism by which varicocele impairs fertility in affected men remains unknown (24), but increased oxidative stress has been suggested to be involved (26).

Oxidative stress is a potent mechanism that may lead to sperm damage, deformity, and ultimately male infertility (27). Increased reactive oxygen species (ROS) levels have also been correlated with decreased sperm motility (28). In the present study, it was identified that the protein expression of TRPC5 was significantly decreased in patients with varicocele-associated asthenozoospermia. Thus, the downregulation of TRPC5 in spermatozoa may correlate with enhanced oxidative stress, which would further adversely affect sperm motility, as observed in the current patient cohort. Although the exact role of TRPC5 in sperm function is yet to be determined, it may be reasoned that the anti-oxidative stress role of TRPC5 contributes to sperm motility. As the key enzyme for scavenging ROS, SOD is the most predominant enzymatic antioxidant in spermatozoa and may prevent reduction in sperm motility (27). As predicted, the present data demonstrated that sperm SOD activity was decreased in the patients with varicocele-associated asthenozoospermia, indicating ongoing oxidative stress in the patients. Furthermore, it was also identified that SOD activity was positively correlated with sperm TRPC5 protein expression. Collectively these results indicate that TRPC5 may serve an important role in sperm motility. However, the exact role of TRPC5 in varicocele-associated asthenozoospermia and how TRPC5 affects sperm motility are yet to be elucidated.

In conclusion, the current study demonstrated the presence of TRPC5 localized in the flagellum and head of human sperm and that sperm TRPC5 levels were significantly decreased in patients with varicocele-associated asthenozoospermia. Furthermore, sperm TRPC5 levels were positively correlated with sperm motility. Therefore, TRPC5 may serve an important role in sperm motility. The present study may provide a novel target for the study and treatment of varicocele-associated asthenozoospermia. However, a limitation is the lack of verification in an animal model; therefore, establishment of animal models and application of corresponding specific agonists should be performed in future studies.

\section{Acknowledgements}

Not applicable.

\section{Funding}

The current study was supported by the Heilongjiang Province Health and Family Planning Commission of Science Foundation of China (grant no. 2017-440).

\section{Availability of data and materials}

All data generated or analyzed during this study are included in this published article.

\section{Authors' contributions}

ZY and YW analyzed and interpreted the patient data. GZ, CX, DC and XW performed the experiments. GZ and CX were primarily responsible for the writing of the manuscript. All authors read and approved the final manuscript.

\section{Ethics approval and consent to participate}

Informed consent was obtained from subjects permitting the use of their samples and data for the current study purposes. The study protocol was approved by the Ethics Committee of Zhongnan Hospital of Wuhan University (Wuhan, China).

\section{Consent for publication}

Informed consent was obtained from subjects permitting the publication of relevant data. 


\section{Competing interests}

The authors declare that they have no competing interests.

\section{References}

1. Darszon A, Labarca P, Nishigaki T and Espinosa F: Ion channels in sperm physiology. Physiol Rev 79: 481-510, 1999.

2. Kumar PG and Shoeb M: The role of trp ion channels in testicular function. Adv Exp Med Biol 704: 881-908, 2011.

3. Cox $\mathrm{T}$ and Peterson RN: Identification of calcium conducting channels in isolated boar sperm plasma membranes. Biochem Biophys Res Commun 161: 162-168, 1989.

4. Nilius B: TRP channels in disease. Biochim Biophys Acta 1772: 805-812, 2007.

5. Voets T, Talavera K, Owsianik G and Nilius B: Sensing with TRP channels. Nat Chem Biol 1: 85-92, 2005.

6. Wes PD, Chevesich J, Jeromin A, Rosenberg C, Stetten G and Montell C: TRPC1, a human homolog of a Drosophila store-operated channel. Proc Natl Acad Sci USA 92: 9652-9656, 1995.

7. Zhu X, Jiang M, Peyton M, Boulay G, Hurst R, Stefani E and Birnbaumer L: trp, a novel mammalian gene family essential for agonist-activated capacitative $\mathrm{Ca}^{2+}$ entry. Cell 85: 661-671, 1996.

8. Clapham DE: TRP channels as cellular sensors. Nature 426 : 517-524, 2003.

9. Montell C: Physiology, phylogeny, and functions of the TRP superfamily of cation channels. Sci STKE 2001: re1, 2001.

10. Putney JW Jr: The enigmatic TRPCs: Multifunctional cation channels. Trends Cell Biol 14: 282-286, 2004.

11. Sutton KA, Jungnickel MK, Wang Y, Cullen K, Lambert S and Florman HM: Enkurin is a novel calmodulin and TRPC channel binding protein in sperm. Dev Biol 274: 426-435, 2004.

12. Treviño CL, Serrano CJ, Beltrán C, Felix R and Darszon A: Identification of mouse trp homologs and lipid rafts from spermatogenic cells and sperm. FEBS Lett 509: 119-125, 2001.

13. Castellano LE, Treviño CL, Rodríguez D, Serrano CJ, Pacheco J, Tsutsumi V, Felix R and Darszon A: Transient receptor potential (TRPC) channels in human sperm: Expression, cellular localization and involvement in the regulation of flagellar motility. FEBS Lett 541: 69-74, 2003.

14. World Health Organization: WHO Manual for the Standardised Investigation and Diagnosis of Infertile Couple. Cambridge University Press, Cambridge, UK, p10, 2000.
15. Dunson DB, Baird DD and Colombo B: Increased infertility with age in men and women. Obstet Gynecol 103: 51-56, 2004.

16. Sharlip ID, Jarow JP, Belker AM, Lipshultz LI, Sigman M, Thomas AJ, Schlegel PN, Howards SS, Nehra A, Damewood MD, et al: Best practice policies for male infertility. Fertil Steril 77: 873-882, 2002.

17. Kamischke A and Nieschlag E: Varicocele treatment in the light of evidence-based andrology. Hum Reprod Update 7: 65-69, 2001.

18. World Health Organization: Laboratory Manual for the Examination and Processing of Human Semen and Sperm-Cervical Mucus Interaction. 5th edition. Cambridge University Press, Cambridge, UK, pp8-9, 2010.

19. Parrish JJ, Krogenaes A and Susko-Parrish JL: Effect of bovine sperm separation by either swim-up or Percoll method on success of in vitro fertilization and early embryonic development. Theriogenology 44: 859-869, 1995.

20. He DX, Gu XT, Jiang L, Jin J and Ma X: A methylation-based regulatory network for microRNA 320a in chemoresistant breast cancer. Mol Pharmacol 86: 536-547, 2014.

21. Darszon A, Beltrán C, Felix R, Nishigaki T and Treviño CL: Ion transport in sperm signaling. Dev Biol 240: 1-14, 2001.

22. Ho HC and Suarez SS: Hyperactivation of mammalian spermatozoa: Function and regulation. Reproduction 122: 519-526, 2001.

23. Okada T, Shimizu S, Wakamori M, Maeda A, Kurosaki T, Takada N, Imoto K and Mori Y: Molecular cloning and functional characterization of a novel receptor-activated TRP $\mathrm{Ca}^{2+}$ channel from mouse brain. J Biol Chem 273: 10279-10287, 1998.

24. French DB, Desai NR and Agarwal A: Varicocele repair: Does it still have a role in infertility treatment? Curr Opin Obstet Gynecol 20: 269-274, 2008.

25. Naughton CK, Nangia AK and Agarwal A: Pathophysiology of varicoceles in male infertility. Hum Reprod Update 7: 473-481, 2001.

26. Marmar JL: The pathophysiology of varicoceles in the light of current molecular and genetic information. Hum Reprod Update 7: 461-472, 2001.

27. Makker K, Agarwal A and Sharma R: Oxidative stress \& male infertility. Indian J Med Res 129: 357-367, 2009.

28. Armstrong JS, Rajasekaran M, Chamulitrat W, Gatti P,Hellstrom WJ and Sikka SC: Characterization of reactive oxygen species induced effects on human spermatozoa movement and energy metabolism. Free Radic Biol Med 26: 869-880, 1999. 